PROCEEDINGS OF THE

AMERICAN MATHEMATICAL SOCIETY

Volume 127, Number 9, Pages 2625-2632

S 0002-9939(99)04932-1

Article electronically published on April 23, 1999

\title{
EXPANDING A BAND
}

\author{
GORDON MACDONALD
}

(Communicated by Palle E. T. Jorgensen)

\begin{abstract}
Any pure operator band can be expanded so that each component
of the band is reflexive.
\end{abstract}

\section{INTRODUCTION}

Recently, there has been an investigation into the structure of semigroups of idempotent operators. This investigation began when H. Radjavi [7] showed that any semigroup of idempotent operators acting on a finite dimensional space is reducible (has an invariant subspace).

Since then, there have been a number of advances in understanding the structure of semigroups of idempotent operators acting on infinite-dimensional spaces (see [2], $[3],[4])$.

Two recent breakthroughs highlight the complexity of such structures. In [5], it has been shown that in the absence of topology, all semigroups of idempotents are algebraically reducible. That is, every idempotent semigroup of linear transformations acting on a vector space has an invariant linear manifold. However, in [1], a construction is given of a semigroup of bounded idempotent operators, acting on a Hilbert space, which has no closed invariant subspace (is irreducible).

Advances in the structure theory of semigroups of bounded idempotent operators have led to a number of results concerning reducibility in some special cases. In this paper, we shall contribute further to the structure theory for semigroups of bounded idempotent operators and establish reducibility in more cases.

The classical theory of idempotent semigroups is quite well established, and one of the interesting facets of the theory of semigroups of bounded idempotent operators is how the classical abstract concepts manifest themselves in the concrete case where the idempotent semigroup is represented as operators.

Before examining some of the classical theory and its implications in the operator case, let us review the basic definitions and terminology involved.

An operator acting on a Hilbert space $H$ is a bounded linear transformation from $H$ to $H$ and the space of all operators acting on a Hilbert space is denoted by $B(H)$. A semigroup is a set closed under some associative binary operation, and by a semigroup of operators we shall mean a subset of $B(H)$ which is closed under multiplication. An idempotent is an element $x$ of a semigroup such that

Received by the editors March 12, 1997 and, in revised form, December 2, 1997.

1991 Mathematics Subject Classification. Primary 20M20, 47D03; Secondary 47A15.

Key words and phrases. Semigroup, idempotent, band, reducible, reflexive.

The author thanks NSERC Canada for their support.

(C)1999 American Mathematical Society 
$x^{2}=x$, and an idempotent operator is an operator $E$ such that $E^{2}=E$. A semigroup of idempotents is usually referred to as a band, so we shall refer to a semigroup of idempotent operators as an operator band. A set of operators $\mathcal{S}$ is reducible if there exists a nontrivial proper subspace (closed in the Hilbert space topology) which is left invariant by every operator in $\mathcal{S}$.

The study of bands has a long history, and a survey of what is known can be found in [6]. However, the study of operator bands (or the representation theory of bands) is very young (see [3] and [4]). The aforementioned three papers contain many of the basic facts which I will state (without proof) about the basic structure of operator bands. These facts shall be used extensively in what follows.

There is a natural preorder defined on a band $\mathcal{S}$. Given $x, y \in \mathcal{S}$, we say $x \preccurlyeq y$ if $x y x=x$. This preorder gives rise to an equivalence relation $\approx$, where given $x, y \in \mathcal{S}$, $x \approx y$ if $x \preccurlyeq y$ and $y \preccurlyeq x$. This equivalence relation is actually a congruence relation, that is, if for $x, y, w, z \in \mathcal{S}$ we have $x \approx y$ and $w \approx z$, then $x w \approx y z$. Thus, $\mathcal{S} / \approx$ (the set of equivalence classes) is also a band and it is easily checked that this is a commutative band and inherits the preorder from $\mathcal{S}$ which becomes a partial order on $\mathcal{S} / \approx$ (and makes $\mathcal{S} / \approx$ a meet semilattice). We shall refer to congruence classes as components and refer to $\mathcal{S} / \approx$ as the band of components. The components have the special property that given any three elements $x, y$ and $z$ of a component, $x y z=x z$. In the case where we have an operator band, the components can be realized concretely. Fix a distinguished member $E$ of a component and write its $2 \times 2$ operator block matrix relative to the decomposition $H=E H+(I-E) H$, so

$$
E=\left[\begin{array}{ll}
I & 0 \\
0 & 0
\end{array}\right]
$$

Then every other member $F$ of the same component has a matrix (relative to the above decomposition) of the form

$$
F=\left[\begin{array}{ll}
I & X_{F} \\
Y_{F} & Y_{F} X_{F}
\end{array}\right]
$$

where not only does $X_{F} Y_{F}=0$, but since for $F$ and $G$ in the component, $E F$ and $G E$ are also in the component and $(E F)(G E)=E$ so $X_{F} Y_{G}=0$. That is, the $(1,2)$ entry of any member of the component times the $(2,1)$ entry of any other member of the component is 0 .

As mentioned above, there is now an example of an irreducible operator band. However, there are many conditions known which force a band to be reducible. If a band $\mathcal{S}$ has a minimal element (a nonzero element $E \in \mathcal{S}$ such that there is no other nonzero nonequivalent element $F \in \mathcal{S}$ with $F \preccurlyeq E$ ), then $\mathcal{S}$ is reducible. Also, if the span of a band $\mathcal{S}$ (the subspace of all linear combinations of operators in $\mathcal{S}$ ) in $B(H)$ contains a compact operator, then $\mathcal{S}$ is reducible (see [4] for the proofs of these results).

In keeping with the terminology of [2], we shall call an operator band a pure band if the the band of components is totally ordered. In [3] it is shown that on finite dimensional spaces, pure bands are just principal-ideal bands and a canonical form is developed in that case. The irreducible band exhibited in [1] is pure (its lattice of components has order type $\omega^{*}$ ) so purity alone is not enough to guarantee reducibility.

In this paper we shall show that any pure band is contained in a larger pure band in which each of the components are reflexive (in the sense that any idempotent $S$ 
such that $S(x) \in \overline{\operatorname{span}\left\{\mathcal{S}_{\alpha}(x)\right\}}$ (where $\mathcal{S}_{\alpha}$ is a component of $\mathcal{S}$ ) is contained in $\mathcal{S}_{\alpha}$. This new band will often have the property that its span contains many compact operators. Thus, appealing to the result of [4] mentioned above, the larger band will be reducible, so the original one will be as well.

\section{Structure of a pure Band}

Our first step is to get a basic understanding of the structure of pure bands. Let $\mathcal{A}$ and $\mathcal{B}$ be two components of an operator band $\mathcal{S}$ in $B(H)$ with $\mathcal{A} \preccurlyeq \mathcal{B}$. For any $A \in \mathcal{A}$ there exists $B \in \mathcal{B}$ such that $A B=B A=A$. (Just choose any element of $\mathcal{B}$ and premultiply and postmultiply by $A$ to get $B$.) Consider the decomposition $H=A H \dot{+}(B-A) H \dot{+}(I-B) H$ and for each $S$ in $\mathcal{S}$ let $\left[S_{i j}\right]_{i j=1}^{3}$ denote the $3 \times 3$ block operator matrix of $S$ with respect to this decomposition. Then using facts about the component structure mentioned above, we have that

$$
A=\left[\begin{array}{lll}
I & 0 & 0 \\
0 & 0 & 0 \\
0 & 0 & 0
\end{array}\right]
$$

and any other element of $\mathcal{A}$ has form

$$
\left[\begin{array}{lll}
I & A_{12} & A_{13} \\
A_{21} & A_{21} A_{12} & A_{21} A_{13} \\
A_{31} & A_{31} A_{12} & A_{31} A_{13}
\end{array}\right] .
$$

Similarly,

$$
B=\left[\begin{array}{lll}
I & 0 & 0 \\
0 & I & 0 \\
0 & 0 & 0
\end{array}\right]
$$

and any other element of $\mathcal{B}$ has form

$$
\left[\begin{array}{lll}
I & 0 & B_{13} \\
0 & I & B_{23} \\
B_{31} & B_{32} & B_{31} B_{13}+B_{32} B_{23}
\end{array}\right] .
$$

Set

$$
\mathcal{A}_{i j}=\left\{A_{i j}: A=\left[A_{i j}\right]_{i j=1}^{3} \in \mathcal{A}\right\}
$$

for $i=1$ and $j=2$ or 3 or $j=1$ and $i=2$ or 3 , and

$$
\mathcal{B}_{i j}=\left\{B_{i j}: B=\left[B_{i j}\right]_{i j=1}^{3} \in \mathcal{B}\right\}
$$

for $i=3$ and $j=1$ or 2 or $j=3$ and $i=1$ or 2 . Then, using the above terminology, we have the following lemmas.

Lemma 2.1. For $\mathcal{A}_{i j}\left(i=1\right.$ and $j=2$ or 3 or $j=1$ and $i=2$ or 3 ) and $\mathcal{B}_{i j}$ (for $i=3$ and $j=1$ or 2 or $j=3$ and $i=1$ or 2$)$ defined as above, we have that:

(a) $\mathcal{A}_{12} \mathcal{A}_{21}=0$ and $\mathcal{A}_{13} \mathcal{A}_{31}=0$.

(b) $\mathcal{B}_{i 3} \mathcal{B}_{3 j}=0$ for $i, j=1$ or 2 .

(c) $\mathcal{B}_{13} \subseteq \mathcal{A}_{13}$ and $\mathcal{B}_{31} \subseteq \mathcal{A}_{31}$.

(d) $\mathcal{A}_{12} \mathcal{B}_{23} \subseteq \operatorname{span} \mathcal{A}_{13}, \mathcal{A}_{13} \mathcal{B}_{32} \subseteq \operatorname{span} \mathcal{A}_{12}, \mathcal{B}_{23} \mathcal{A}_{31} \subseteq \operatorname{span} \mathcal{A}_{21}$ and $\mathcal{B}_{32} \mathcal{A}_{21} \subseteq$ $\operatorname{span} \mathcal{A}_{31}$. 
Proof. Let $A$ and $B$ be idempotents as above. To prove (a) consider $E$ and $F$ in $\mathcal{A}$. Then $E B F$ and $E F$ are in $\mathcal{A}$ so $A E B F A=A$ and $A E F A=A$. Thus, writing the $3 \times 3$ matrices for these idempotents with respect to the above decomposition of $H$ and multiplying:

$$
\left[\begin{array}{lll}
I & 0 & 0 \\
0 & 0 & 0 \\
0 & 0 & 0
\end{array}\right]=A=A E B F A=\left[\begin{array}{lll}
I+E_{12} F_{12} & 0 & 0 \\
0 & 0 & 0 \\
0 & 0 & 0
\end{array}\right]
$$

and

$$
\left[\begin{array}{lll}
I & 0 & 0 \\
0 & 0 & 0 \\
0 & 0 & 0
\end{array}\right]=A=A E F A=\left[\begin{array}{lll}
I+E_{12} F_{12}+E_{13} F_{13} & 0 & 0 \\
0 & 0 & 0 \\
0 & 0 & 0
\end{array}\right]
$$

so $E_{12} F_{12}=0$ and $E_{12} F_{12}+E_{13} F_{13}=0$ so $E_{13} F_{13}=0$. Since $E$ and $F$ were arbitrary elements in $\mathcal{A}$, (a) follows.

To prove (b) consider $G$ and $H$ in $\mathcal{B}$. Then $G H$ is in $\mathcal{B}$ so $B G H B=B$. Writing the $3 \times 3$ matrices for these idempotents and multiplying them gives

$$
\left[\begin{array}{ccc}
I & 0 & 0 \\
0 & I & 0 \\
0 & 0 & 0
\end{array}\right]=B=B G H B=\left[\begin{array}{lll}
I+G_{13} H_{31} & G_{13} H_{32} & 0 \\
G_{23} H_{31} & I+G_{23} H_{32} & 0 \\
0 & 0 & 0
\end{array}\right]
$$

so $G_{i 3} H_{3 j}=0$ for $i, j=1$ or 2 since $G$ and $H$ were arbitrary elements of $\mathcal{B}$, (b) follows.

To prove (c) consider $G$ in $\mathcal{B}$. Then $A G$ and $G A$ are in $\mathcal{A}$ so $(A G)_{13}=G_{13} \subseteq \mathcal{A}_{13}$ and $(G A)_{31}=G_{31} \subseteq \mathcal{A}_{31}$. Since $G$ was an arbitrary element of $\mathcal{B}$, (c) follows.

To prove (d), consider $E$ in $\mathcal{A}$ and $G$ in $\mathcal{B}$. Now $A E B G$ is in $\mathcal{A}$ so

$$
A E B G=\left[\begin{array}{lll}
I & 0 & G_{13}+E_{12} G_{23} \\
0 & 0 & 0 \\
0 & 0 & 0
\end{array}\right] \in \mathcal{A}
$$

so $G_{13}+E_{12} G_{23} \in \mathcal{A}_{13}$. By (c), $G_{13} \in \mathcal{A}_{13}$ so $E_{12} G_{23} \in \operatorname{span} \mathcal{A}_{13}$.

Similarly, by considering $A E G B, B G E A$ and $G B E A$ the other inclusions follow.

There is almost a converse to Lemma 2.1.

Lemma 2.2. Given any decomposition $H=M_{1} \dot{+} M_{2} \dot{+} M_{3}$ and subspaces $\mathcal{A}_{i j} \subseteq$ $B\left(M_{j}, M_{i}\right)$ (for $i=1$ and $j=2$ or 3 or $j=1$ and $i=2$ or 3 ) and $\mathcal{B}_{i j} \subseteq$ $B\left(M_{j}, M_{i}\right)$ (for $i=3$ and $j=1$ or 2 or $j=3$ and $i=1$ or 2 ) which satisfy the conditions

(a) $\mathcal{A}_{12} \mathcal{A}_{21}=0$ and $\mathcal{A}_{13} \mathcal{A}_{31}=0$.

(b) $\mathcal{B}_{i 3} \mathcal{B}_{3 j}=0$ for $i, j=1$ or 2 .

(c) $\mathcal{B}_{13} \subseteq \mathcal{A}_{13}$ and $\mathcal{B}_{31} \subseteq \mathcal{A}_{31}$.

(d) $\mathcal{A}_{12} \mathcal{B}_{23} \subseteq \mathcal{A}_{13}, \mathcal{A}_{13} \mathcal{B}_{32} \subseteq \mathcal{A}_{12}, \mathcal{B}_{23} \mathcal{A}_{31} \subseteq \mathcal{A}_{21}$ and $\mathcal{B}_{32} \mathcal{A}_{21} \subseteq \mathcal{A}_{31}$. 
Then, with respect to the above decomposition of $H$, the operators

$$
\begin{aligned}
& \left\{\left[\begin{array}{lll}
I & A_{12} & A_{13} \\
A_{21} & A_{21} A_{12} & A_{21} A_{13} \\
A_{31} & A_{31} A_{12} & A_{31} A_{13}
\end{array}\right]: A_{i j} \in \mathcal{A}_{i j}\right\} \\
& \cup\left\{\left[\begin{array}{lll}
I & 0 & B_{13} \\
0 & I & B_{23} \\
B_{31} & B_{32} & B_{31} B_{13}+B_{32} B_{23}
\end{array}\right]: B_{i j} \in \mathcal{B}_{i j}\right\}
\end{aligned}
$$

form a pure band.

Proof. We just need to check that this set is closed under multiplication and consists of idempotents. Both of these are routine matrix calculations. So this is a band. It is also pure since we have not added any new components, except possibly for the zero component.

The previous Lemmas are similar to Theorems 2 and 3 of [3] which describe in more detail how the components of a Principal-ideal band fit together in the finitedimensional case. With these two lemmas at our disposal we are almost ready to state and prove the main theorem of the paper.

\section{EXPANDING A PURE BAND}

As mentioned previously, given a pure band, we wish to construct a new band in such a way that, hopefully, the new band will contain numerous compacts in its span. The mechanics of how we do this are the crux of this section. First, we need one more piece of terminology.

Definition 3.1. If $\mathcal{S}$ is a subset of $B(H)$, then $\operatorname{ref}(\mathcal{S})$ is defined to be all operators $T$ in $B(H)$ such that for any $x \in H, T(x) \in \overline{\operatorname{span}\{S(x): S \in \mathcal{S}\}}$ (the closed linear span) and $\operatorname{ref}_{\text {idempotent }}(\mathcal{S})$ is defined to be all idempotent operators $T$ in $B(H)$ such that for any $x \in H, T(x) \in \overline{\operatorname{span}\{S(x): S \in \mathcal{S}\}}$.

The definition of $\operatorname{ref}_{\text {idempotent }}(\mathcal{S})$ is an obvious translation of the definition of $\operatorname{ref}(\mathcal{S})$ to the case where the only operators under consideration are idempotent. The definition of $\operatorname{ref}(\mathcal{S})$ itself is a generalization of a concept from operator algebras. Given a unital subalgebra $\mathcal{A}$ of $B(H)$, lat $\mathcal{A}$ is defined to be the lattice of all subspaces of $H$ which are invariant under every operator in $\mathcal{A}$, and given a lattice of subspaces $\mathcal{L}, \operatorname{alg} \mathcal{L}$ denotes the algebra of all operators which leave all the subspaces in $\mathcal{L}$ invariant. Since, for unital algebras $\mathcal{A}$, lat $\mathcal{A}$ is generated by the cyclic invariant subspaces of $\mathcal{A}$, we have that alg lat $\mathcal{A}=\operatorname{ref}(\mathcal{A})$. An algebra $\mathcal{A}$ which satisfies alg lat $\mathcal{A}=\mathcal{A}$ is called reflexive, and so a subspace $\mathcal{S}$ which satisfies $\operatorname{ref}(\mathcal{S})=\mathcal{S}$ is called reflexive. (See $[8]$ for more information on reflexive subspaces.)

We shall sometimes write $\overline{\operatorname{span}\{\mathcal{S}(x)\}}$ for $\overline{\operatorname{span}\{S(x): S \in \mathcal{S}\}}$ and when we write $\mathcal{L} \mathcal{M}$ where $\mathcal{L}$ and $\mathcal{M}$ are subsets of $B(H)$ we mean all possible products of elements of $\mathcal{L}$ times elements of $\mathcal{M}$. The following two lemmas contain crucial facts we will need to know about ref.

Lemma 3.2. Let $\mathcal{L}, \mathcal{M}$ and $\mathcal{N}$ be subsets of $B(H)$ and $\mathcal{L M} \subseteq \mathcal{N}$; then

$$
\operatorname{ref}(\mathcal{L}) \operatorname{ref}(\mathcal{M}) \subseteq \operatorname{ref}(\mathcal{N}) .
$$


Proof. If $\mathcal{L} \mathcal{M} \subseteq \mathcal{N}$ and $L \in \operatorname{ref}(\mathcal{L})$ and $M \in \operatorname{ref}(\mathcal{M})$, then

$$
\begin{aligned}
L M(x) & =L(M(x)) \in \overline{\operatorname{span}\{\mathcal{L} M(x)\}} \\
& \subseteq \overline{\operatorname{span}\{\mathcal{L}(\overline{\operatorname{span}\{\mathcal{M}(x)\}})\}} \\
& \subseteq \overline{\operatorname{span}\{\mathcal{L M}(x)\} \subseteq \overline{\operatorname{span}\{\mathcal{N}(x)\}}}=\operatorname{ref}(\mathcal{N})
\end{aligned}
$$

so $\operatorname{ref}(\mathcal{L}) \operatorname{ref}(\mathcal{M}) \subseteq \operatorname{ref}(\mathcal{N})$.

Lemma 3.3. If $\mathcal{L}$ is a subset of $B(H)$ and $P$ and $Q$ are operators in $B(H)$, then

$$
\operatorname{Pref}(\mathcal{L}) Q \subseteq \operatorname{ref}(P \mathcal{L} Q)=\operatorname{ref}(\{P L Q: L \in \mathcal{L}\}) .
$$

Proof. If $M \in \operatorname{ref}(\mathcal{L})$ and $x \in H$, then

$$
P M Q(x)=P(M(Q x)) \in \overline{P \operatorname{span}\{\mathcal{L} Q(x)\}} \subseteq \overline{\operatorname{span}\{P \mathcal{L} Q(x)\}}
$$

so $P M Q \in \operatorname{ref}(P \mathcal{L} Q)$.

The following lemma computes $\operatorname{ref}_{\text {idempotent }}(\mathcal{S})$ in the special case of a onecomponent band.

Lemma 3.4. If

$$
\mathcal{S}=\left\{\left[\begin{array}{ll}
I & X \\
Y & Y X
\end{array}\right]: X \in \mathcal{X} \text { and } Y \in \mathcal{Y}\right\}
$$

is a one-component band contained in $B(H)$, then

$$
\operatorname{ref}_{\text {idempotent }}(\mathcal{S})=\left\{\left[\begin{array}{ll}
I & U \\
V & V U
\end{array}\right]: U \in \operatorname{ref}(\mathcal{X}) \text { and } V \in \operatorname{ref}(\mathcal{Y})\right\} \cup\left\{\left[\begin{array}{ll}
0 & 0 \\
0 & 0
\end{array}\right]\right\}
$$

Proof. If $C \in \operatorname{ref}_{\text {idempotent }}(\mathcal{S})$ has block matrix

$$
C=\left[\begin{array}{ll}
C_{11} & C_{12} \\
C_{21} & C_{22}
\end{array}\right]
$$

with respect to the same decomposition of $H$ as used for $\mathcal{S}$ above, then

$$
\begin{aligned}
{\left[\begin{array}{ll}
C_{11} & C_{12} \\
C_{21} & C_{22}
\end{array}\right]\left[\begin{array}{l}
x \\
0
\end{array}\right] } & =C\left[\begin{array}{l}
x \\
0
\end{array}\right] \in \overline{\operatorname{span}\left\{S\left[\begin{array}{l}
x \\
0
\end{array}\right]: S \in \mathcal{S}\right\}} \\
& =\frac{\operatorname{span}\left\{\left[\begin{array}{ll}
I & X \\
Y & Y X
\end{array}\right]\left[\begin{array}{l}
x \\
0
\end{array}\right]: X \in \mathcal{X} \text { and } Y \in \mathcal{Y}\right\}}{\operatorname{span}\left\{\left[\begin{array}{ll}
I & X \\
Y & Y X
\end{array}\right]\left[\begin{array}{l}
x \\
0
\end{array}\right]: X \in \mathcal{X} \text { and } Y \in \mathcal{Y}\right\}} \\
& =\frac{\operatorname{span}\left\{\left[\begin{array}{l}
I(x) \\
Y(x)
\end{array}\right]: Y \in \mathcal{Y}\right\}}{}
\end{aligned}
$$

so $C_{11} \in \operatorname{ref}(I)=\lambda I$ and $C_{21} \in \operatorname{ref}(\mathcal{Y})$. Similarly, one can show that $C_{22} \in \operatorname{ref}(\mathcal{Y} \mathcal{X})$, and by looking at orthogonal complements, one can show that $C_{12} \in \operatorname{ref}(\mathcal{X})$. By the previous lemma, since $\mathcal{X Y}=0$, we have that $C_{12} C_{21}=0$ and $\left(C_{22}\right)^{2}=0$. Since $C$ is idempotent, this implies that $\left(C_{11}\right)^{2}=C_{11}$ so $\lambda=0$ or 1 .

If $\lambda=0$, then

$$
\left[\begin{array}{ll}
0 & C_{12} \\
C_{21} & C_{22}
\end{array}\right]=C=C^{2}=\left[\begin{array}{ll}
0 & C_{12} \\
C_{21} & C_{22}
\end{array}\right]^{2}=\left[\begin{array}{ll}
0 & C_{12} C_{22} \\
C_{22} C_{21} & C_{21} C_{12}
\end{array}\right]
$$


so $C_{12}=C_{12} C_{22}=C_{12} C_{21} C_{12}=0$ and similarly, $C_{21}=0$ so $C_{22}=0$ which means that $C=0$.

If $\lambda=1$, then

$$
\left[\begin{array}{ll}
I & C_{12} \\
C_{21} & C_{22}
\end{array}\right]=C=C^{2}=\left[\begin{array}{ll}
I & C_{12} \\
C_{21} & C_{22}
\end{array}\right]^{2}=\left[\begin{array}{ll}
I & C_{12}+C_{12} C_{22} \\
C_{21}+C_{22} C_{21} & C_{21} C_{12}
\end{array}\right]
$$

so $C_{22}=C_{21} C_{12}$ and the result follows.

We are now ready to state and prove our main theorem.

Theorem 3.5. Let $\mathcal{S}$ be a pure band with components $\mathcal{S}(\alpha)$ for $\alpha$ in $A=\mathcal{S} / \approx($ so $\mathcal{S}=\bigcup_{\alpha \in A} \mathcal{S}(\alpha)$ ) and let $\mathcal{T}(\alpha)=\operatorname{ref}_{\text {idempotent }}(\mathcal{S})$. Then $\mathcal{T}=\bigcup_{\alpha \in A} \mathcal{T}(\alpha)$ is a pure band containing $\mathcal{S}$.

Proof. We need to show that $\mathcal{T}$ is closed under multiplication. Once this is established, since $\mathcal{T}$ is defined as a set of idempotents, and the only new component possibly created in this construction is the zero component, $\mathcal{T}$ will be a pure band. To that end, let $T \in \mathcal{T}(\alpha)$ and $S \in \mathcal{T}(\beta)$ with $\alpha \leq \beta$. We will show that $T S$ and $S T$ are in $\mathcal{T}(\alpha)$. If either $T$ or $S$ is zero, this is obviously true so assume otherwise.

Choose $A \in \mathcal{S}(\alpha)$ and $B \in \mathcal{S}(\beta)$ such that $A B=B A=A$ and consider the decomposition $H=A H \dot{+}(B-A) H \dot{+}(I-B) H$. By Lemma 3.3, (using the notation of Lemma 2.1), since $T$ is in $\mathcal{T}(\alpha)$, with respect to this decomposition of $H$,

$$
T=\left[\begin{array}{lll}
I & T_{12} & T_{13} \\
T_{21} & T_{21} T_{12} & T_{21} T_{13} \\
T_{31} & T_{31} T_{12} & T_{31} T_{13}
\end{array}\right]
$$

where

$$
\left[\begin{array}{cc}
T_{12} & T_{13}
\end{array}\right] \in \operatorname{ref}\left(\mathcal{X}_{\alpha}\right)
$$

and

$$
\left[\begin{array}{l}
T_{21} \\
T_{31}
\end{array}\right] \in \operatorname{ref}\left(\mathcal{Y}_{\alpha}\right)
$$

Similarly, since $S$ is in $\mathcal{T}(\beta)$,

$$
S=\left[\begin{array}{lll}
I & 0 & S_{13} \\
0 & I & S_{23} \\
S_{31} & S_{32} & S_{31} S_{13}+S_{32} S_{23}
\end{array}\right]
$$

where

$$
\left[\begin{array}{l}
S_{13} \\
S_{23}
\end{array}\right] \in \operatorname{ref}\left(\mathcal{X}_{\beta}\right)
$$

and

$$
\left[\begin{array}{ll}
S_{31} & S_{32}
\end{array}\right] \in \operatorname{ref}\left(\mathcal{Y}_{\beta}\right)
$$

Hence, by Lemma 3.2 (taking $P$ or $Q$ to be $A$ or $(B-A)$ ) we obtain that $T_{12} \in$ $\operatorname{ref}\left(\mathcal{A}_{12}\right), T_{13} \in \operatorname{ref}\left(\mathcal{A}_{13}\right), T_{21} \in \operatorname{ref}\left(\mathcal{A}_{21}\right)$ and $T_{31} \in \operatorname{ref}\left(\mathcal{A}_{31}\right)$ and $S_{13} \in \operatorname{ref}\left(\mathcal{B}_{13}\right)$, $S_{23} \in \operatorname{ref}\left(\mathcal{B}_{23}\right), S_{31} \in \operatorname{ref}\left(\mathcal{B}_{31}\right)$ and $S_{32} \in \operatorname{ref}\left(\mathcal{B}_{32}\right)$.

By Lemma 2.1 and Definition 3.1, the subspaces $\operatorname{ref}\left(\mathcal{A}_{i j}\right)(i=1$ and $j=2$ or 3 or $j=1$ and $i=2$ or 3 ) and $\operatorname{ref}\left(\mathcal{B}_{i j}\right)$ (for $i=3$ and $j=1$ or 2 or $j=3$ and $i=1$ or 2) satisfy the hypotheses of Lemma 2.2 , and hence $S T$ and $T S$ are idempotent. It 
is also a consequence of Lemma 2.2 that $E S T E=E, E T S E=E, S T E S T=S T$ and $T S E T S=T S$ for all $E$ in $\mathcal{T}(\alpha)$. Also,

$$
\begin{aligned}
S T(x) & \in \overline{\operatorname{span}\{\mathcal{S}(\beta) T(x)\}} \subseteq \overline{\operatorname{span}\{\mathcal{S}(\beta) \overline{\operatorname{span}\{\mathcal{S}(\alpha)(x)\}}\}} \\
& \subseteq \overline{\operatorname{span}\{\mathcal{S}(\beta) \mathcal{S}(\alpha)(x)\}} \subseteq \overline{\operatorname{span}\{\mathcal{S}(\alpha)(x)\}}
\end{aligned}
$$

for all $x$ in $H$ so $S T \in \operatorname{ref}(\mathcal{S}(\alpha))$ and similarly $T S \in \operatorname{ref}(\mathcal{S}(\alpha))$. Thus, $S T$ and $T S$ satisfy all three conditions for membership in $\mathcal{T}(\alpha)=\operatorname{ref}_{\text {idempotent }}(\mathcal{S})$ and the theorem is proven.

Theorem 3.5 has the following consequence for reducibility.

Theorem 3.6. If any component

$$
\mathcal{S}(\alpha)=\left\{\left[\begin{array}{ll}
I & X \\
Y & Y X
\end{array}\right]: X \in \mathcal{X}_{\alpha} \text { and } Y \in \mathcal{Y}_{\alpha}\right\}
$$

of a pure band $\mathcal{S}$ contained in $B(H)$ is such that $\operatorname{ref}\left(\mathcal{X}_{\alpha}\right)$ or $\operatorname{ref}\left(\mathcal{Y}_{\alpha}\right)$ contains a compact operator, then $\mathcal{S}$ is reducible.

Proof. This follows directly from Theorem 3.5, Lemma 3.4 and [4] wherein it states that any band containing a compact operator in its span is reducible.

\section{REFERENCES}

1. R. Drnovšek, An irreducible semigroup of idempotents, Studia Math. 125 (1997), 97-99. MR 98e: 47011

2. P. Fillmore, G. MacDonald, M. Radjabalipour and H. Radjavi, Towards a classification of maximal unicellular pure bands, Semigroup Forum 49 (1994), 195-215. MR 95f: 47059

3. P. Fillmore, G. MacDonald, M. Radjabalipour and H. Radjavi, Principal-Ideal Bands, Semigroup Forum, to appear.

4. L. Livshits, G. MacDonald, B. Mathes and H. Radjavi, Reducible semigroups of idempotent operators, J. Operator Theory, 40 (1998), 35-69.

5. L. Livshits, G. MacDonald, B. Mathes and H. Radjavi, Do bands have non-trivial irreducible representations?, submitted for publication.

6. M. Petrich, Lectures in Semigroups, Berlin:Academie-Verlag/John Wiley \& Sons Ltd., 1977. MR 56:5749; MR 57:6150

7. H. Radjavi, On the reduction and triangularization of semigroups of operators, J. Operator Theory 13 (1985), 65-71. MR 86c:47056

8. A. Loginov and V. Sulman, hereditary and intermediate reflexivity of $W^{*}$-algebras, Izv. Akad. Nauk SSSR, 39 (1975), 1260-1273; USSR-Isv., 9 (1975). MR 53:8919

Department of Mathematics and Computer Science, University of Prince Edward

Island, Charlottetown, Prince Edward Island, Canada C1A 4P3

E-mail address: gmacdonald@upei.ca 